\title{
Independent effects of circulating glucose, insulin and NEFA on cardiac triacylglycerol accumulation and myocardial insulin resistance in a swine model
}

\author{
Maria Angela Guzzardi • Leanne Hodson • Letizia Guiducci • Elena Sanguinetti • \\ Pietro Di Cecco • Tiziana Liistro • Cristina Vassalle • Silvia Pardini • Lucia Giorgetti • \\ Piero A. Salvadori • Silvia Burchielli • Patricia Iozzo
}

Received: 11 February 2014 / Accepted: 19 May 2014 / Published online: 25 June 2014

(C) Springer-Verlag Berlin Heidelberg 2014

\begin{abstract}
Aims/hypothesis Cardiac steatosis and myocardial insulin resistance elevate the risk of cardiac complications in obesity and diabetes. We aimed to disentangle the effects of circulating glucose, insulin and NEFA on myocardial triacylglycerol (TG) content and myocardial glucose uptake.

Methods Twenty-two pigs were stratified according to four protocols: low NEFA+low insulin (nicotinic acid), high NEFA+low insulin (fasting) and high insulin+low NEFA \pm high glucose (hyperinsulinaemia-hyperglycaemia or hyperinsulinaemia-euglycaemia). Positron emission tomography, $\left[\mathrm{U}-{ }^{13} \mathrm{C}\right]$ palmitate enrichment techniques and tissue biopsies were used to assess myocardial metabolism. Heart rate and rate-pressure product (RPP) were monitored.

Results Myocardial glucose extraction was increased by NEFA suppression and was similar in the hyperinsulinaemiahypergylcaemia, hyperinsulinaemia-euglycaemia and nicotinic acid groups. Hyperglycaemia enhanced myocardial glucose
\end{abstract}

M. A. Guzzardi • L. Guiducci • E. Sanguinetti $\cdot$ T. Liistro •

S. Pardini $\cdot$ P. A. Salvadori $\cdot$ P. Iozzo $(\square)$

Institute of Clinical Physiology, National Research Council (CNR),

Via Moruzzi 1, 56124 Pisa, Italy

e-mail: patricia.iozzo@iff.cnr.it

L. Hodson

Oxford Centre for Diabetes, Endocrinology and Metabolism

(OCDEM), University of Oxford, Oxford, UK

E. Sanguinetti $\cdot$ T. Liistro

Scuola Superiore di Studi Universitari Sant'Anna, Pisa, Italy

P. Di Cecco $\cdot$ C. Vassalle $\cdot$ S. Burchielli

Fondazione Toscana Gabriele Monasterio (FTGM), Pisa, Italy

L. Giorgetti

Institute of Agricultural Biology and Biotechnology, National

Research Council (CNR), Pisa, Italy uptake due to a mass action. Myocardial TG content was greatest in the fasting group, whereas hyperinsulinaemia had a mild effect. Heart rate and RPP increased in hyperinsulinaemia-euglycaemia, in which cardiac glycogen content was reduced. Heart rate correlated with myocardial TG and glycogen content.

Conclusions/interpretation Elevated NEFA levels represent a powerful, self-sufficient promoter of cardiac TG accumulation and are a downregulator of myocardial glucose uptake, indicating that the focus of treatment should be to 'normalise' adipose tissue function to lower the risk of cardiac TG accumulation and myocardial insulin resistance. The observation that hyperinsulinaemia and nicotinic acid led to myocardial fuel deprivation provides a potential explanation for the cardiovascular outcomes reported in recent intensive glucoselowering and NEFA-lowering clinical trials.

Keywords Cardiac steatosis · Fatty acid metabolism . Glucose metabolism · Insulin resistance $\cdot$ Positron emission tomography

\begin{tabular}{ll}
\multicolumn{2}{l}{ Abbreviations } \\
DBP & Diastolic blood pressure \\
${ }^{18}$ F-FDG & {$\left[{ }^{18}\right.$ F $]$ fluoro-2-deoxyglucose } \\
FAME & Fatty acid methyl ester \\
G-Rd & Rate of disappearance of glucose \\
GU & Glucose uptake \\
H\&E & Haematoxylin and eosin \\
HR & Heart rate \\
MPE & Mole percentage excess \\
$3-O H B$ & $\beta$-Hydroxybutyrate \\
PAS & Periodic acid-Schiff \\
PET & Positron emission tomography \\
Ra & Rate of appearance
\end{tabular}




\section{RPP Rate-pressure product \\ SBP Systolic blood pressure \\ TG Triacylglycerol \\ TTR Tracer-to-tracee ratio}

low and high plasma NEFA concentrations and hyperinsulinaemia on myocardial insulin sensitivity, fatty acid metabolism and the synthesis and content of TG.

\section{Introduction}

Myocardial fat accumulation and myocardial insulin resistance have been implicated in the pathogenesis of cardiac complications in obesity and diabetes. A progressive increase in myocardial fat content has been observed in obese and type 2 diabetic patients [1], and has been shown to correlate with a decline in left ventricular function and signs of cardiomyopathy $[2,3]$. Increased myocardial triacylglycerol (TG) storage may reflect an imbalance between the supply and utilisation of fatty acids. In animal models, fatty acid overflow contributes to the development of diastolic dysfunction, promoting cardiac hypertrophy and fibrosis [4-7]. An elevation of circulating NEFA, as is sometimes seen in obesity and type 2 diabetes, is associated with myocardial insulin resistance, in which the persistent use of NEFA can lead to an impaired consumption of glucose, in line with the Randle cycle, and inefficient ATP production $[8,9]$. As a consequence of increasing NEFA levels and insulin resistance, there is an upregulation of the pathways involved in fatty acid metabolism, while glucose metabolism is inhibited [10]. In these conditions, cardiac work and oxygen utilisation are enhanced [11], hampering the response of the myocardium to hormonal stimuli and ischaemia.

Several studies have suggested that the modulation of myocardial TG by suppression of plasma substrate (i.e. NEFA and glucose) and hormone (i.e. insulin) levels may be beneficial. However, the relative contribution of hyperglycaemia, elevated levels of plasma NEFA and hyperinsulinaemia to the regulation of ectopic lipid accumulation and myocardial insulin sensitivity remains unclear. Although a positive association between hyperglycaemia and myocardial TG has been reported with obesity, impaired glucose tolerance and type 2 diabetes [1], the induction of short-term hyperglycaemia by insulin deprivation in type 1 diabetes did not change myocardial TG content or left ventricle function [12]. An elevation in plasma NEFA, resulting from short-term caloric restriction, starvation or an acute bout of exercise in healthy and/or type 2 diabetic individuals, has been associated with myocardial TG accumulation and decreased left ventricle diastolic function [2, 13-15]. Moreover, the pharmacological suppression of circulating NEFA by the antilipolytic nicotinic acid derivative acipimox prevented the accumulation of myocardial TG during exercise [14]. Conversely, plasma NEFA elevation after 3 days of high-fat feeding did not affect myocardial function or TG accumulation [16]. The purpose of the current study was to investigate the independent roles of hyperglycaemia,

\section{Methods}

Study design The study design is schematised in Fig. 1. Twenty-two weight-matched pigs $(29.6 \pm 1.0 \mathrm{~kg}$, mean \pm SEM) were fasted for 14-15 $\mathrm{h}$ before the study. Each protocol consisted of a stabilisation period ( $t=-120$ to $t=0 \mathrm{~min})$ during which $\left[\mathrm{U}_{-}{ }^{13} \mathrm{C}\right]$ palmitate complexed to albumin $\left(0.04 \mu \mathrm{mol} \mathrm{kg}^{-1} \mathrm{~min}^{-1}\right)$ was infused to achieve steady-state enrichment. From $t=0$ to $t=300 \mathrm{~min}$, the following protocols were applied: (1) hyperinsulinaemia-hyperglycaemia $(n=6)$, in which insulin was infused at a prime-constant rate of $1 \mathrm{mU} \mathrm{kg}^{-1} \mathrm{~min}^{-1}$ and glucose was co-administered to achieve a glycaemia of approximately $15 \mathrm{mmol} / 1$; (2) hyperinsulinaemia-euglycaemia $(n=6)$ in which euglycaemia was maintained; (3) fasting $(n=5)$, in which saline $(0.9 \%)$ was infused, resulting in a prolonged, i.e. approximately $20 \mathrm{~h}$, fasting period at the time of imaging and tissue sampling; and (4) low NEFA, in which nicotinic acid $(n=5)$ was administered at a rate of $1.5 \mathrm{mg} \mathrm{kg}^{-1} \mathrm{~min}^{-1}$ [17]. In the fasting and nicotinic groups, saline was used to match the infusion volumes of protocols 1 and 2.

The experimental protocol was conducted in accordance with the D.L. 116/92 implementation of the directive EEC $609 / 86$ regarding the protection of animals used for experimental and other scientific purposes.

Animal preparation and positron emission tomography scanning The animals were sedated on the morning of the study with tiletamine and zolazepam $(10 \mathrm{mg} / \mathrm{kg}$ i.m. Zoletil; Virbac Laboratories, Carros, France), and anaesthesia was maintained by an infusion of pentothal sodium $(8-12 \mathrm{ml} / \mathrm{h}$, $40 \mathrm{mg} / \mathrm{ml}$; MSD Animal Health, Milan, Italy). The animals were mechanically ventilated via tracheal intubation with oxygen and normal room air (fraction of inspired oxygen $30-40 \%$, respiratory volume $100-150 \mathrm{ml} \mathrm{kg}^{-1} \mathrm{~min}^{-1}, 16$ breaths $/ \mathrm{min}$ ). Catheters were placed in the following sites: in the femoral veins for the infusion of glucose (dextrose $20 \%$ ), insulin (Abbott Laboratories, Chicago, IL, USA), $\left[\mathrm{U}_{-}{ }^{13} \mathrm{C}\right]$ palmitate (Cambridge Isotopes, CK Gas Products, Cambridge, UK), nicotinic acid (Sigma-Aldrich, St Louis, $\mathrm{MO}$, USA) and saline; in the ear veins for $\left[{ }^{18} \mathrm{~F}\right]$ fluoro-2deoxyglucose $\left({ }^{18} \mathrm{~F}-\mathrm{FDG}\right)$ injections and anaesthetic infusions; in a femoral artery for blood sampling; and in the bladder to quantify ${ }^{18} \mathrm{~F}$-FDG urinary losses in a subset of animals.

The animals were transported to the positron emission tomography (PET) facility and positioned supine with the thoracic area in the gantry of an ECAT HR+ tomograph 


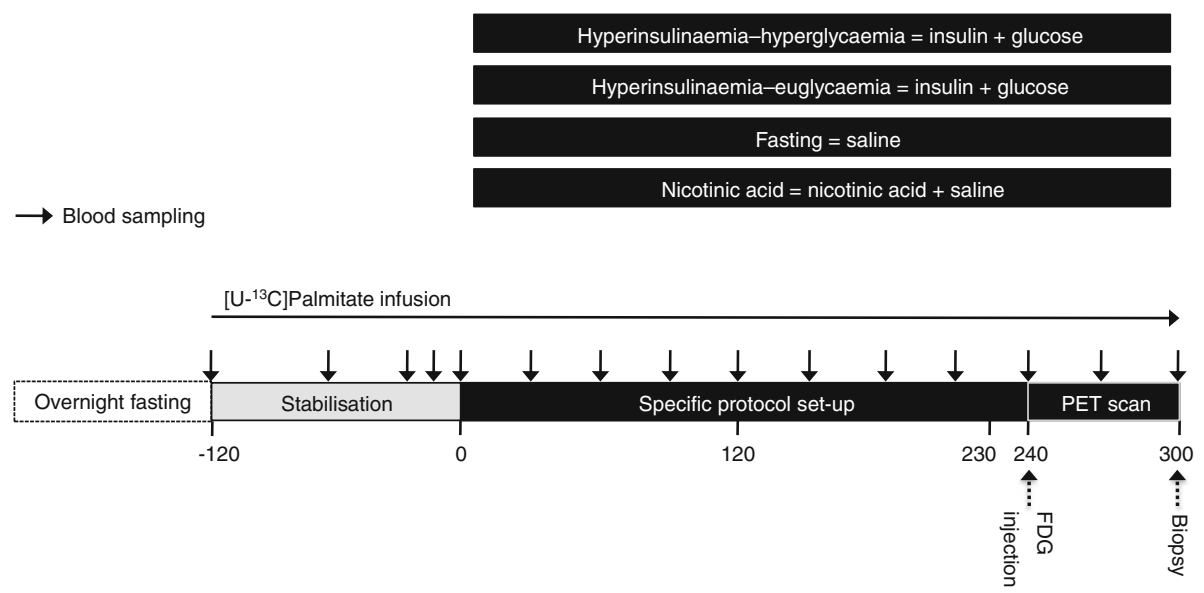

Fig. 1 The study design. The white block represents the overnight fasting period. The grey block indicates the first step of the experimental protocol, during which $\left[\mathrm{U}_{-}{ }^{13} \mathrm{C}\right]$ palmitate infusion was started and the metabolic and cardiac variables were allowed to stabilise after instrumentation. The black bars at the top of the diagram represent the time course

(Siemens CTI, Knoxville, TN, USA). The infusion protocols were started, and at $t=230 \mathrm{~min}$ a transmission scan was performed to measure photon attenuation. At $t=240 \mathrm{~min}$, the glucose analogue ${ }^{18} \mathrm{~F}-\mathrm{FDG}(284 \pm 15 \mathrm{MBq}, \mathrm{mean} \pm \mathrm{SEM})$ was administered by rapid i.v. injection and a 60 min dynamic scan was started. Arterial blood samples were taken every $30 \mathrm{~min}$ for the measurement of plasma glucose, TG, cholesterol, NEFA, $\beta$-hydroxybutyrate (3-OHB) and $\left[\mathrm{U}-{ }^{13} \mathrm{C}\right]$ palmitate enrichment in the NEFA and TG pools as previously described $[18,19]$. Vital signs, haematocrit, blood pressure and heart rate (HR) were monitored throughout the study. The animals were killed with an overdose of sodium pentobarbital. The chest was opened and myocardial tissue samples were collected and flash-frozen in liquid nitrogen.

Image processing All sinograms were corrected for deadtime, decay and photon attenuation and were reconstructed by standard algorithms. Regions of interest were drawn on images corresponding to three consecutive planes of the left ventricle to obtain myocardial ${ }^{18}$ F-FDG time-activity curves, and at the base of the left ventricular chamber of the heart to obtain corresponding input functions, representing the amount of tracer available in the circulation for organ extraction over time. The curves were corrected for residual spillover and partial volume effects [20]. In addition, input functions were corrected for the competitive effect of plasma glucose [21].

Systemic and myocardial glucose metabolism Graphical analysis [22] was used to quantify the myocardial glucose clearance $\left(K \mathrm{i}, \mathrm{ml} \mathrm{min}^{-1} \mathrm{ml}^{-1}\right)$. Myocardial glucose uptake (GU; $\mu \mathrm{mol} \mathrm{min}^{-1} \mathrm{ml}^{-1}$ ) was obtained by multiplying the $K \mathrm{i}$ values by the steady-state plasma glucose concentrations and dividing by a lumped constant term of $1.0[23,24]$. of the four infusion protocols, each lasting $5 \mathrm{~h}$, including $1 \mathrm{~h}$ of PET acquisition. The time scale is given below the blocks. Solid arrows indicate blood withdrawals; dotted arrows indicate FDG injection and the collection of tissue biopsy samples at the end of the study

The systemic glucose disappearance rates (G-Rds; $\mu \mathrm{mol} \mathrm{min} \mathrm{mg}^{-1}$ ) were calculated according to a previously validated method [25]. The injected ${ }^{18} \mathrm{~F}-\mathrm{FDG}$ dose was discounted for the average $20 \%$ of ${ }^{18} \mathrm{~F}-\mathrm{FDG}$ found in the urine, as measured in a subset of animals. The resulting dose was divided by the integral of plasma activity from $t=0$ to $t=\infty$ to obtain the metabolic glucose clearance rate. G-Rd was estimated as the product of the clearance rate and the steadystate glycaemia.

Systemic and myocardial $\left[\mathrm{U}_{-}{ }^{13} \mathrm{C}\right]$ palmitate metabolism Total lipids were extracted from tissue and plasma [26]. Tissue and plasma TG and plasma NEFA were separated from the other lipid fractions by solid-phase extraction [27] and the prepared fatty acid methyl esters (FAMEs) were analysed by gas chromatography [28]. Unlabelled plasma palmitate concentrations were calculated by multiplying the proportion of palmitate in the plasma lipid pool by the corresponding plasma concentrations of NEFA or TG determined enzymatically [19].

$\left[\mathrm{U}-{ }^{13} \mathrm{C}\right]$ palmitate enrichments in the FAME derivatives of NEFA and TG were analysed by GC-MS [19]. Tracer-totracee ratios (TTRs) for $\left[\mathrm{U}_{-}{ }^{13} \mathrm{C}\right]$ palmitate $(\mathrm{M}+16) /(\mathrm{M}+0)$ were calculated. The baseline measurement (before administration of the stable isotope tracer) was subtracted from the TTR of each sample (both plasma and tissue) to allow for natural abundance. The TTRs were multiplied by the corresponding palmitate NEFA or palmitate TG concentrations to give plasma or tissue tracer concentrations. The rate of appearance $\left(\mathrm{Ra} ; \mu \mathrm{mol} \mathrm{min}{ }^{-1} \mathrm{~kg}^{-1}\right)$ of NEFA was calculated by dividing the $\left[\mathrm{U}_{-}^{13} \mathrm{C}\right]$ palmitate infusion rate by the plasma NEFA enrichment (measured as mole percentage excess [MPE]), under the assumption that the Ra is equal to the rate of disappearance. The myocardial palmitate esterification rate 
was calculated as ratio of TG $\left[\mathrm{U}_{-}{ }^{13} \mathrm{C}\right]$ palmitate TTR in the myocardium to the integrated plasma NEFA and TG $\left[\mathrm{U}-{ }^{13} \mathrm{C}\right]$ palmitate TTRs, multiplied by the concentration of total unlabelled palmitate in both plasma pools.

Myocardial TG content Samples ( 100 mg) of myocardial tissue (left ventricle) were homogenised in absolute ethanol. After $2 \mathrm{~h}$ of shaking at room temperature, the cell debris was allowed to settle and the supernatant fraction was separated by centrifugation at 2,000 $\mathrm{g}$ for $5 \mathrm{~min}$ at room temperature. Myocardial TG content was measured by an enzymatic assay using a commercial kit (Giesse Diagnostic, Rome, Italy).

Cardiac histology, glycogen and lipid content Cryosections, $8 \mu \mathrm{m}$ thick and fixed in cold $10 \%$ neutral buffered formalin, were stained with haematoxylin and eosin (H\&E) and the Periodic acid-Schiff (PAS) reaction (Bio-Optica, Milan, Italy) according to standard protocols, to evaluate tissue morphology and glycogen content, respectively. Stained sections were acquired using an optical microscope connected to a colour camera (Carl Zeiss, Oberkochen, Germany). The glycogen content was assessed using a semi-quantitative grading scale of staining intensity with a score ranging from 1 (low intensity) to 3 (high intensity). For each sample, a score was calculated as the mean value of three independent determinations of randomly selected and blindly examined sections.

Cardiac workload Resting HR and systolic and diastolic blood pressure (SBP, DBP) were monitored over time, and their dependence on the infusion protocol was examined. The rate-pressure product $(\mathrm{RPP}=\mathrm{SBP} \times \mathrm{HR})$ was used as index of cardiac workload.

Statistical analysis Data were analysed using SPSS for MAC (version 20; SPSS, Chicago, IL, USA). Results are expressed as the median (range) and analysed by the non-parametric Wilcoxon and Mann-Whitney tests, for paired or independent samples. Spearman's correlation coefficients were used to determine associations. Multivariate regression analysis was used to establish independent relationships. A $p$ value $<0.05$ was considered to be significant.

\section{Results}

The metabolic variables of the four groups are summarised in Table 1.

At steady state, the insulin levels were higher in hyperinsulinaemia-hypergylcaemia and hyperinsulinaemiaeuglycaemia groups compared with the fasting and nicotinic acid groups, and higher in the hyperinsulinaemiahyperglycaemia than the hyperinsulinaemia-euglycaemia group. Plasma NEFA levels decreased in the hyperinsulinaemia-hypergylcaemia, nicotinic acid and hyperinsulinaemia-euglycaemia groups, with the change being most notable in the latter $(p=0.028)$; no decrease was noted in the fasting group. The decline in plasma TG was significant in hyperinsulinaemia-euglycaemia, consistent with a suppression of hepatic VLDL-TG production. The higher plasma TG concentrations observed in the hyperinsulinaemia-hyperglycaemia group may be explained by hepatic de novo lipogenesis, as indicated by a significant increase in lipogenic index of the plasma TGs (16:0/18:2-6, $p<0.05$ ); a change in lipogenic index was not observed in the hyperinsulinaemia-euglycaemia group.

Plasma 3-OHB levels tended to increase over time in the fasting group $(p=0.08)$ and decrease in the hyperinsulinaemia-euglycaemia group $(p=0.028)$. The change in 3-OHB was associated with the change in NEFA levels $(r=0.588, p=0.013)$.

Systemic and myocardial glucose metabolism The variables relating to systemic glucose metabolism are summarised in Table 2. No significant changes in glucose, insulin and glucose infusion rates occurred after $2 \mathrm{~h}$, indicating steady state. Systemic glucose clearance and G-Rd were higher in the hyperinsulinaemia-hyperglycaemia group and progressively decreased in the hyperinsulinaemia-euglycaemia group, fasting and nicotinic acid groups. G-Rd was correlated with steady-state insulin concentrations $(r=0.861, p<0.001)$.

Myocardial Ki reflects the intrinsic response of the heart to glucose extraction. It was lowest in the fasting group, reaching significance vs the hyperinsulinaemia-euglycaemia and nicotinic acid groups (Fig. 2a). Myocardial GU incorporates Ki and the influence of ambient glycaemia (glucose mass action), both of which determine the uptake of glucose by the heart. Myocardial GU was highest in the hyperinsulinaemiahyperglycaemia, followed by the hyperinsulinaemiaeuglycaemia and then the nicotinic acid and fasting groups; it was more than two times higher in the nicotinic acid than the fasting group (Fig. 2b, c). Myocardial GU was positively correlated with glucose $(r=0.554, p=0.009)$ and insulin $(r=$ $0.652, p=0.001)$ levels, and inversely associated with NEFA concentrations $(r=-0.589, p=0.006)$ and the increment in plasma 3-OHB $(r=-0.626, p=0.004)$. In multiple regression analysis, myocardial glucose clearance was independently related to NEFA levels (inversely, $p=0.057$ ) but not to insulinaemia $(p=0.92)$ or glycaemia $(p=0.46)$.

Systemic and myocardial $\left[\mathrm{U}_{-}{ }^{13} \mathrm{C}\right]$ palmitate and TG metabolism The systemic and myocardial palmitate data are summarised in Table 2. The plasma Ra of NEFA was higher in the fasting compared with the hyperinsulinaemiaeuglycaemia group $(p=0.045)$. It was positively correlated with plasma NEFA levels $(r=0.397, p=0.05)$, and inversely 


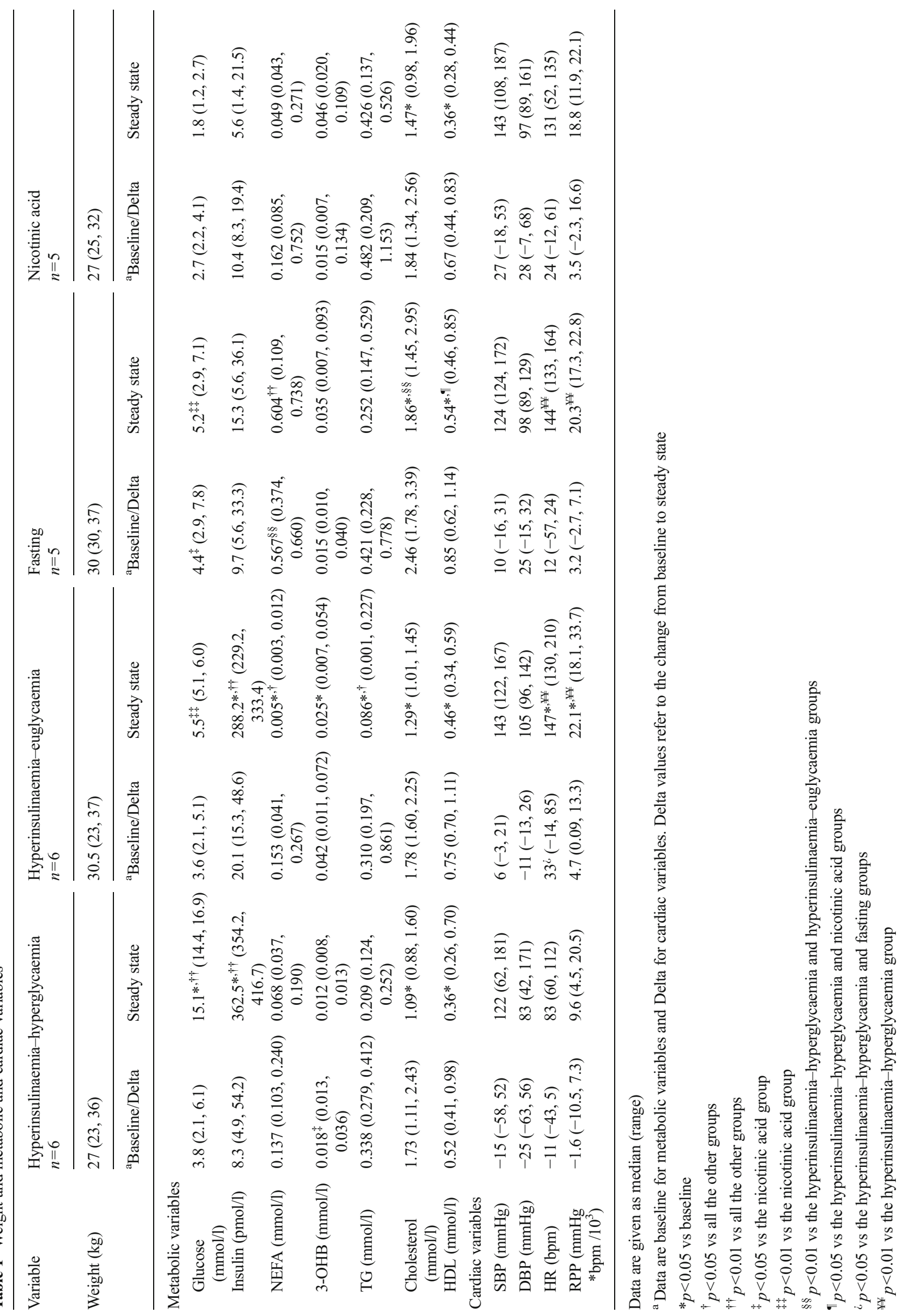


Table 2 Systemic metabolism and myocardial palmitate esterification

\begin{tabular}{|c|c|c|c|c|}
\hline Variable & $\begin{array}{l}\text { Hyperinsulinaemia- } \\
\text { hyperglycaemia } \\
n=6\end{array}$ & $\begin{array}{l}\text { Hyperinsulinaemia- } \\
\text { euglycaemia } \\
n=6\end{array}$ & $\begin{array}{l}\text { Fasting } \\
n=5\end{array}$ & $\begin{array}{l}\text { Nicotinic acid } \\
n=5\end{array}$ \\
\hline Glucose clearance $\left(\mathrm{ml} \mathrm{min}^{-1} \mathrm{~kg}^{-1}\right)$ & $45.7 * *(14,79)$ & $18.5^{*}(7.6,31)$ & $6.0(5.1,16)$ & $4.8(2.5,8.3)$ \\
\hline G-Rd $\left(\mu \mathrm{mol} \mathrm{min} \mathrm{mg}^{-1} \mathrm{~kg}^{-1}\right)$ & $660^{\dagger \dagger}(224,1,131)$ & $98 *(40,177)$ & $33(16,81)$ & $7.8^{\dagger \dagger}(2.7,23)$ \\
\hline NEFA Ra $\left(\mu \mathrm{mol} \mathrm{min}{ }^{-1} \mathrm{~kg}^{-1}\right)$ & $3.8(1.7,24)$ & $3.6(1.8,11)$ & $11.1^{*}(5.3,32)$ & $7.2(2.1,10)$ \\
\hline $\begin{array}{l}\text { NEFA (palmitate) plasma enrichment } \\
\text { (MPE) }\end{array}$ & $0.055(0.006,0.076)$ & $0.042(0.015,0.072)$ & $0.017(0.009,0.040)$ & $0.030(0.022,0.065)$ \\
\hline $\begin{array}{l}\text { TG (palmitate) plasma enrichment } \\
\text { (MPE) }\end{array}$ & $0.019(0.006,0.026)$ & $0.010(0.005,0.019)$ & $0.008(0.007,0.029)$ & $0.010(0.003,0.046)$ \\
\hline $\begin{array}{l}\text { Myocardial palmitate esterification } \\
\text { rate }\left(\mathrm{nmol} \min ^{-1} \mathrm{~g}^{-1}\right)\end{array}$ & $0.19(0.16,3.93)$ & $0.11(0.01,0.23)$ & $0.65^{\star}(0.20,10.65)$ & $0.10(0.02,1.42)$ \\
\hline
\end{tabular}

Data are given as median (range)

${ }^{*} p<0.05$ vs fasting and nicotinic acid groups

$* * p<0.01$ vs fasting and nicotinic acid groups

${ }^{\dagger \dagger} p<0.01$ vs all the other groups

${ }^{\star} p<0.05$ vs the hyperinsulinaemia-euglycaemia group

associated with myocardial glucose $K \mathrm{i}(r=-0.473, p=0.030)$. Conversely, plasma NEFA $\left[\mathrm{U}_{-}{ }^{13} \mathrm{C}\right]$ palmitate enrichment was significantly lower $(p<0.05)$ in the fasting group compared with both hyperinsulinaemia conditions.

The estimation of myocardial palmitate esterification was based on the assumption that a quasi steady state was reached in the tissue compartment after $7 \mathrm{~h}$ of $\left[\mathrm{U}_{-}{ }^{13} \mathrm{C}\right]$ palmitate infusion as plasma NEFA and TG TTRs achieved steady state in all groups. Myocardial palmitate esterification was higher in the fasting compared with the other groups, with a significantly greater myocardial TG content being noted in the fasting animals (Fig. 3a).

Cardiac histology and glycogen content $\mathrm{H} \& \mathrm{E}$ staining did not show any significant structural difference between the groups. The PAS score, calculated using a semi-quantitative grading scale of staining intensity (Fig. $4 \mathrm{a}$ ) and representing the cardiac glycogen content, was lower in the hyperinsulinaemia-euglycaemia group compared with the other groups (Fig. 4b, d).
Fig. 2 The analysis of myocardial glucose metabolism showed that myocardial glucose clearance $(K \mathrm{~K})$ was reduced in fasting compared with the other conditions (a). Myocardial GU was lower in the fasting group and progressively increased in the nicotinic acid,

hyperinsulinaemia-euglycaemia and hyperinsulinaemia-

hyperglycaemia groups (b), as shown in representative parametric PET images (c). Black circles in (b) represent outliers. $* p<0.05$ a

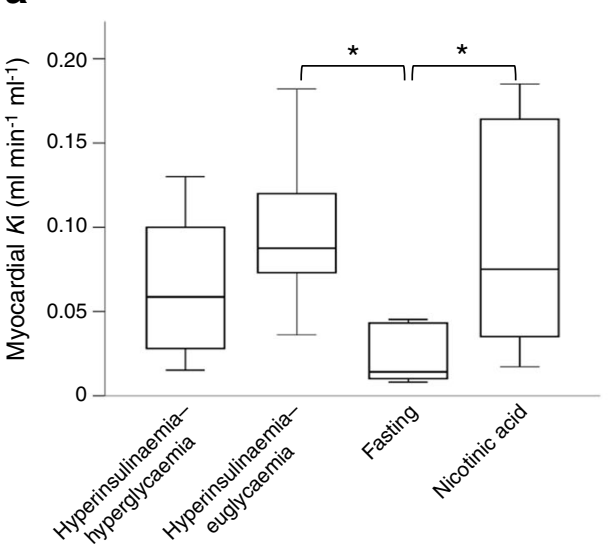

C

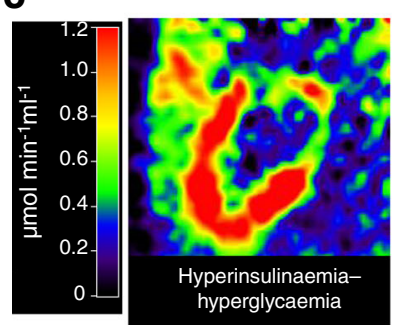

b
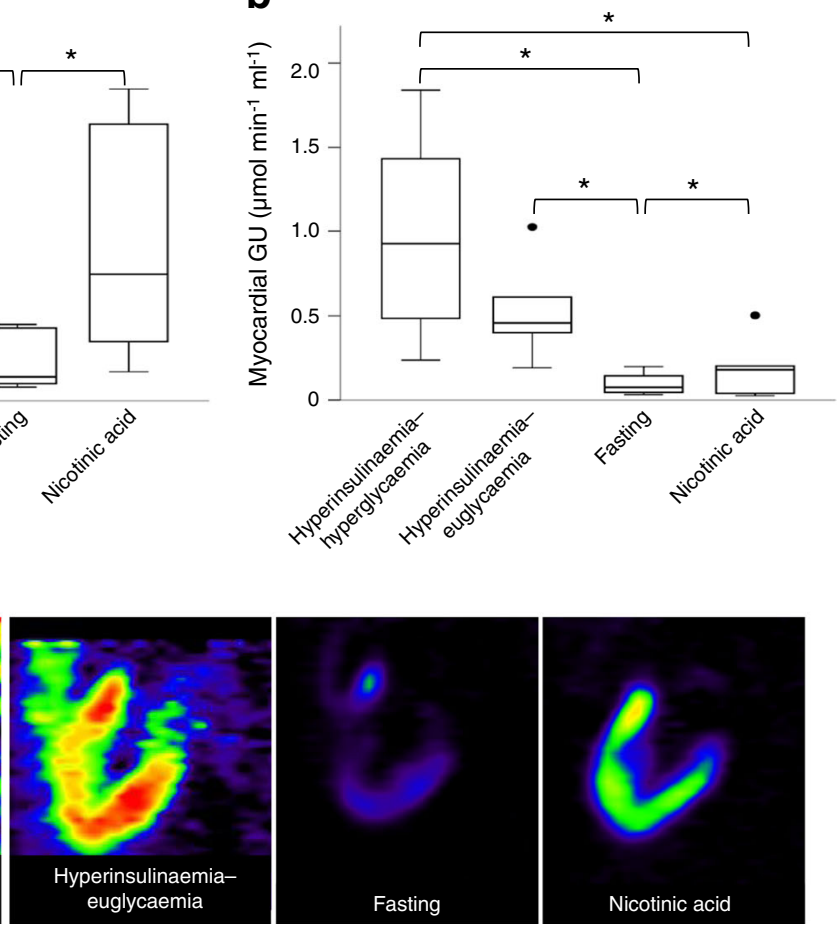
Fig. 3 Myocardial TG content was higher in the fasting group compared with the other groups (a), and correlated with $\mathrm{HR}$ $(p=0.499, r=0.025)$ as an indicator of workload (b) $* p<0.05$ a

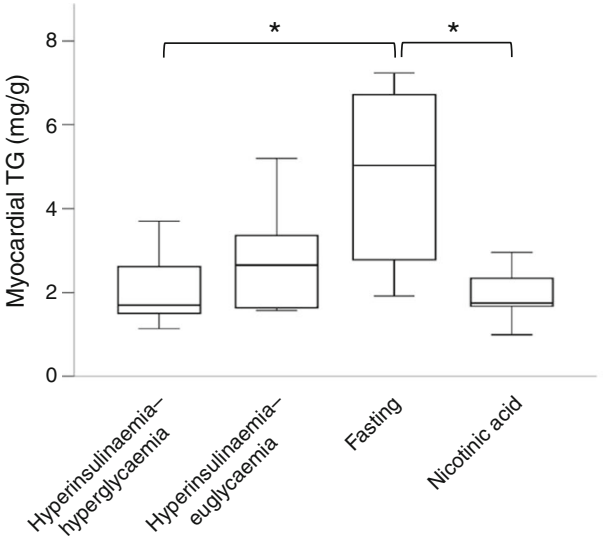

b

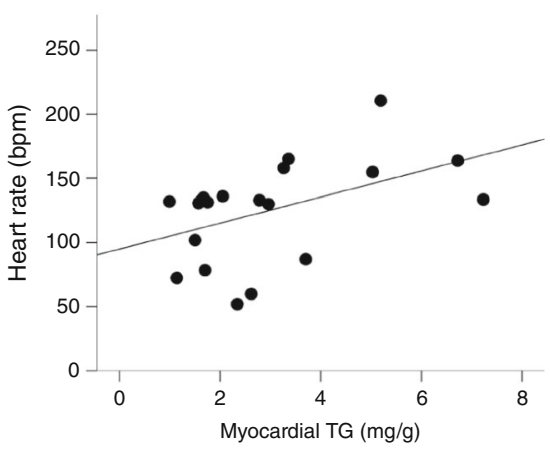

Cardiac workload Changes from resting baseline to steadystate SBP and DBP, HR and RPP are reported in Table 1. No significant differences were observed in blood pressure between the groups during the study. The baseline HR was higher $(p<0.05)$ in the fasting group compared with the hyperinsulinaemia-hyperglycaemia and nicotinic acid groups. HR and RPP increased significantly only in the hyperinsulinaemia-euglycaemia condition. Steady-state RPP tended to be positively associated with myocardial TG and inversely associated with glycogen content. HR was correlated with myocardial TG (Fig. 3b) and glycogen content (Fig. 4c).

\section{Discussion}

Obesity and diabetes are characterised by elevated levels of NEFA, glucose and insulin, which may contribute to the pathogenesis of cardiac steatosis and myocardial insulin resistance, both of which have been implicated in elevating the risk of cardiovascular disease and cardiomyopathy. The purpose of this study was to disentangle the individual roles of glucose, insulin and (physiological) NEFA levels on myocardial glucose and lipid metabolism. Our data demonstrate that plasma NEFA concentrations are of significance in the regulation of myocardial metabolism. Myocardial TG synthesis and content were elevated in response to the supply of NEFA, whereas an inhibition of adipose tissue lipolysis - hormonally or pharmacologically induced - resulted in the promotion of myocardial GU without major changes in myocardial TG accumulation. The effect of insulin on myocardial glucose clearance was likely to be mediated by NEFA suppression, while a considerable (insulin-independent) effect of glucose in promoting myocardial GU was seen.

We used nicotinic acid as a negative control, reflecting the absence of insulin, glucose and NEFA. The prolonged fasting protocol allowed us to examine the isolated effects of
Fig. 4 Myocardial glycogen content, measured using an intensity scale of PAS staining (a), was lower in the hyperinsulinaemia-euglycaemia group with respect to the other groups (b), and was inversely associated with HR ( $p=-0.589$, $r=0.010)$ as an indicator of cardiac workload (c). The differences between the hyperinsulinaemiahyperglycaemia and hyperinsulinaemia-euglycaemia groups are shown $(\mathbf{d})$. Magnification $\times 100$. AU, arbitrary units. ${ }^{*} p<0.05$, $* * p<0.01$ a
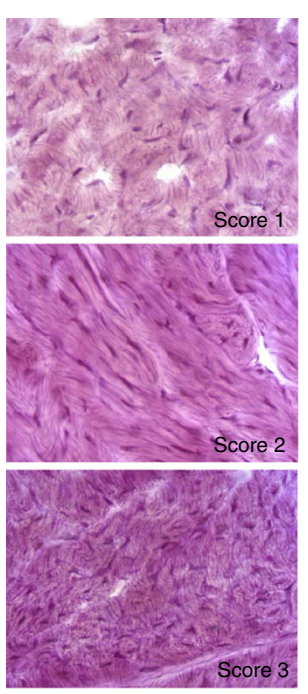

b
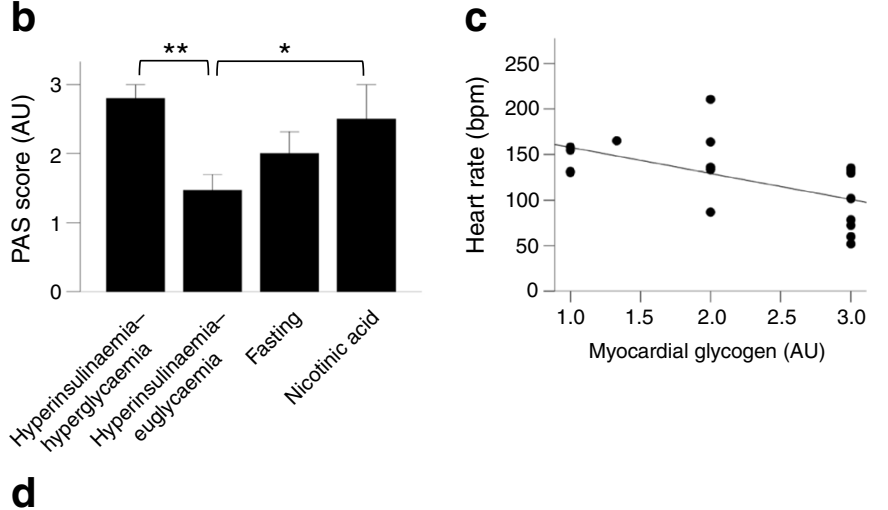

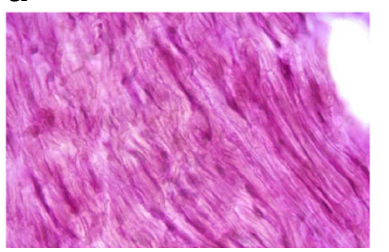

Hyperinsulinaemia-hyperglycaemia

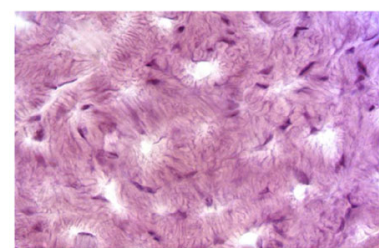

Hyperinsulinaemia-euglycaemia 
physiologically elevated plasma NEFA concentrations. The hyperinsulinaemia-euglycaemia and hyperinsulinaemiahyperglycaemia groups were used to establish the contribution of physiological hyperinsulinaemia and the additional contribution of hyperglycaemia to myocardial TG accumulation and glucose disposal. As a clinical translation, each of the latter three conditions reflects one of the metabolic abnormalities observed in diabetic and obese patients, whereas the nicotinic acid mimicked the metabolic profile that may be achieved with an intensive treatment; both extremes may be detrimental to the metabolism of the heart.

Nicotinic acid administration was associated with low myocardial GU, palmitate esterification and TG content. Previous evidence [29-31] has shown that nicotinic acid enhances myocardial GU by reducing plasma NEFA availability. In our study, the myocardial glucose clearance $(\mathrm{Ki}$, $\mathrm{ml} \mathrm{min}{ }^{-1} \mathrm{ml}^{-1}$; Fig. 2a) was more than twice as high in the nicotinic acid compared with the fasting group, in spite of low insulin and glucose levels, and similar to that observed in the hyperinsulinaemic states. However, myocardial GU (myocardial clearance $\times$ glycaemia; Fig. 2b) in the nicotinic acid group was not as high as in the two hyperinsulinaemia groups, due to the reduction in glycaemia. Although such a decline in glucose levels does not commonly occur in acute studies, we have previously observed a glucose-lowering effect of acipimox in human patients [32]. In the present study, the duration of NEFA suppression was longer than was seen in other acute studies, which may cause a compensatory consumption of glucose by all the body organs, resulting in fuel deprivation. An inefficient compensation of NEFA depletion by glucose or 3-OHB in the myocardium may underlie the reduced cardiac work, stroke volume and/or ejection fraction observed upon acute and chronic acipimox administration in healthy participants $[32,33]$.

Our results indicate that exposure to physiologically elevated NEFA levels may be a key mechanism in promoting TG accumulation in the myocardium, which appears to have a dynamic lipid depot that is responsive to acute metabolic changes [13, 34]. Prolonged fasting $(\sim 20 \mathrm{~h})$ was associated with a rise in plasma NEFA concentrations and appearance rates. Accordingly, $\left[\mathrm{U}_{-}{ }^{13} \mathrm{C}\right]$ palmitate enrichment in this pool was reduced and inversely correlated with NEFA concentrations, consistent with dilution by endogenous NEFA. NEFA entering the liver are partitioned into the ketogenic pathway, as shown by the trend for plasma 3-OHB concentrations to increase. Due to substrate competition [9], systemic and myocardial GU rates were low; the latter was inversely correlated with the increment in 3-OHB and NEFA levels. An increase in myocardial palmitate esterification and content was observed in the fasting group compared with the other groups, a finding consistent with studies in healthy and type 2 diabetic individuals $[2,15,35]$. These studies reported an increased myocardial TG content when lipolysis was induced by caloric restriction or a $48 \mathrm{~h}$ fasting period, unless acipimox was coadministered to counteract increasing NEFA levels [12]. Work in fasting dogs has demonstrated that elevated plasma NEFA concentrations, achieved by intralipid-heparin infusion, were associated with greater content and synthesis rates of myocardial TG compared with conditions with lower plasma NEFA, such as insulin or saline infusion [36]. In contrast, high-fat feeding does not change myocardial TG content in healthy subjects, despite notable increases in plasma TG and NEFA levels and an accumulation of liver fat [16, 35]. Notably, insulin concentrations are low during caloric restriction or intralipid administration, while a high insulin in response to a high-fat diet may limit myocardial TG accumulation by suppressing the release of NEFA from adipose tissue and TG from the liver, which is consistent with our findings.

The more chronic exposure to hyperinsulinaemia that occurs in obese and type 2 diabetes individuals, together with adipose tissue and liver insulin resistance, may result in a more significant effect of insulin on cardiac steatosis. However, our data indicate that the delivery of NEFA to the heart is primarily responsible for myocardial TG accumulation, confirming the latter as a predictor of cardiac workload, as measured by HR. An acute elevation in plasma NEFA levels has been negatively associated with aortic distensibility in healthy individuals [37], supporting the role of plasma NEFA in cardiovascular complications. The elevation of 3-OHB observed in the fasting group may lower the use of glucose and NEFA by the heart, thereby further diverting glycerol and fatty acids into TG synthesis. From a clinical standpoint, NEFA levels are not routinely measured in clinical patients, and our data suggest a need for trials evaluating the predictive value of NEFA for cardiovascular outcomes. The perspective is that NEFA may represent a biomarker that is easily measurable in the clinic, where routine assessments of myocardial steatosis and insulin resistance are not realistic.

Under the hypothesis that glucose represents a source of glycerol for TG synthesis, enhancing the lipogenic effects of hyperinsulinaemia, we investigated the effects of hyperglycaemia-hyperinsulinaemia as a promoter of myocardial TG accumulation. Myocardial glucose clearance was similar in the hyperinsulinaemia-hyperglycaemia and hyperinsulinaemia-euglycaemia groups, suggesting that myocardial insulin sensitivity is superimposable in these two states, whereas it was similar in the nicotinic acid and lower in the fasting group, suggesting that the action of insulin to stimulate myocardial GU is primarily mediated by the suppression of plasma NEFA. This was further supported by multivariate regression data showing an independent association between myocardial glucose clearance and NEFA, but not insulin, levels. Independent of insulin and NEFA, the mass action of glucose can potently enhance its own myocardial uptake, as demonstrated here. However, the increase in myocardial GU did not translate into a significant elevation in 
myocardial TG accumulation compared with that of the hyperinsulinaemic state alone. Hammer et al [38] reported that $24 \mathrm{~h}$ of hyperglycaemia achieved by partial insulin deprivation in patients with type 1 diabetes did not affect the myocardial TG content. In contrast, Winhofer et al [34] demonstrated that combined hyperglycaemia and hyperinsulinaemia induced myocardial lipid accumulation in normal individuals, independent of insulin resistance and while plasma NEFA were suppressed; the authors suggested that insulinaemia rather than glycaemia was responsible. Our data extend this line of evidence by separating the effect of hyperglycaemia from that of hyperinsulinaemia and comparing these with control conditions. We show a modest effect of hyperinsulinaemia on myocardial TG accumulation, which may be explained by the counteracting lack of NEFA.

Our data suggest that, during hyperinsulinaemia, the suppression of circulating NEFA, 3-OHB and TG primarily direct myocardial glucose towards oxidative pathways, consistent with the depletion of myocardial glycogen that was observed in hyperinsulinaemia-euglycaemia. An increased consumption of glycogen may result from an elevation of cardiac workload, as supported by the inverse correlation between glycogen content and HR or RPP. Similarly, in fasting conditions, glycogen depots tended to be lower and RPP was higher. In line with our findings, elevations in HR and RPP have been observed in patients with type 2 diabetes or those fed a high-fat diet $[1,16]$ while no change in haemodynamic variables has been observed following an acute exposure to isolated hyperglycaemia [38] or combined hyperinsulinaemiahyperglycaemia [34].

Some limitations of our study include the sample size. According to ethical committee recommendations, we used the minimum number of animals to guarantee the statistical power of the main endpoints; clear-cut results were achieved during steady state, reducing variability. In order to describe our data appropriately, we performed non-parametric analysis, but the variability in some baseline findings could not be overcome. All the animals were killed at the end of the protocol for tissue collection, so comparisons are across, rather than within, groups for tissue-specific variables. Although we could not directly prove a myocardial steadystate incorporation of ${ }^{13} \mathrm{C}$ - after $7 \mathrm{~h}$ of tracer infusion, steady-state enrichment was reached in the plasma lipid pools before tissue collection.

In conclusion, we have demonstrated, in a controlled fashion, that elevated plasma NEFA concentrations represent a powerful, self-sufficient promoter of myocardial TG accumulation and myocardial insulin resistance. Our data indicate that insulin acts on myocardial glucose clearance primarily by a suppression of NEFA levels. Independent of insulin, hyperglycaemia promotes myocardial GU by mass action. Although reflecting acute phenomena, with an extrapolation to more chronic substrate and hormone exposures being speculative, our work has clinical implications. First, our data suggest that insulin resistance in adipose tissue is an important target for the prevention of the aforementioned cardiac conditions. Second, we have provided evidence that hyperinsulinaemia is a state of partial myocardial fuel deprivation in which glycogen stores may have to be used to support an increase in workload. This evidence may help to explain the negative cardiovascular outcome findings from intensive glucose control clinical trials in patients with diabetes $[39,40]$. Finally, our study supports the implementation of trials examining the predictive power of plasma NEFA levels and of NEFA normalisation on cardiac complications related to obesity and type 2 diabetes.

Acknowledgements M. Gilbert (research technician, Oxford Centre for Diabetes, Endocrinology and Metabolism, OCDEM, University of Oxford, UK) is acknowledged for help with the fatty acid analysis.

Funding The study was supported by IFC-CNR. LH is a British Heart Foundation Intermediate Fellow in Basic Science.

Duality of interest The authors declare that there is no duality of interest associated with this manuscript.

Contribution statement All the authors provided substantial contributions to the conception and design, acquisition and analysis of data. Moreover, all the authors participated in drafting the article and revising it critically for important intellectual content, and gave final approval of the version to be published. PI is responsible for the integrity of the work as a whole.

\section{References}

1. Iozzo P (2009) Viewpoints on the way to the consensus session: where does insulin resistance start? The adipose tissue. Diabetes Care 32(Suppl 2):S168-S173

2. Kankaanpaa M, Lehto HR, Parkka JP et al (2006) Myocardial triglyceride content and epicardial fat mass in human obesity: relationship to left ventricular function and serum free fatty acid levels. J Clin Endocrinol Metab 91:4689-4695

3. Szczepaniak LS, Dobbins RL, Metzger GJ et al (2003) Myocardial triglycerides and systolic function in humans: in vivo evaluation by localized proton spectroscopy and cardiac imaging. Magn Reson Med 49:417-423

4. Guzzardi MA, Iozzo P (2011) Fatty heart, cardiac damage, and inflammation. Rev Diabet Stud 8:403-417

5. Finck BN, Lehman JJ, Leone TC et al (2002) The cardiac phenotype induced by PPARalpha overexpression mimics that caused by diabetes mellitus. J Clin Invest 109:121-130

6. Chiu HC, Kovacs A, Ford DA et al (2001) A novel mouse model of lipotoxic cardiomyopathy. J Clin Invest 107:813-822

7. Lee Y, Naseem RH, Park BH et al (2006) Alpha-lipoic acid prevents lipotoxic cardiomyopathy in acyl CoA-synthase transgenic mice. Biochem Biophys Res Commun 344:446-452

8. Shipp JC, Opie LH, Challoner D (1961) Fatty acid and glucose metabolism in the perfused heart. Nature 189:1018-1019

9. Randle PJ, Garland PB, Hales CN, Newsholme EA (1963) The glucose fatty-acid cycle. Its role in insulin sensitivity and the metabolic disturbances of diabetes mellitus. Lancet 1:785-789 
10. Rider OJ, Cox P, Tyler D, Clarke K, Neubauer S (2013) Myocardial substrate metabolism in obesity. Int J Obes (Lond) 37:972-979

11. Most AS, Brachfeld N, Gorlin R, Wahren J (1969) Free fatty acid metabolism of the human heart at rest. J Clin Invest 48:1177-1188

12. Hammer S, van der Meer RW, Lamb HJ et al (2008) Short-term flexibility of myocardial triglycerides and diastolic function in patients with type 2 diabetes mellitus. Am J Physiol Endocrinol Metab 295:E714-E718

13. Bilet L, van de Weijer T, Hesselink MK et al (2011) Exercise-induced modulation of cardiac lipid content in healthy lean young men. Basic Res Cardiol 106:307-315

14. van der Meer RW, Hammer S, Smit JW et al (2007) Short-term caloric restriction induces accumulation of myocardial triglycerides and decreases left ventricular diastolic function in healthy subjects. Diabetes 56:2849-2853

15. Hammer S, van der Meer RW, Lamb HJ et al (2008) Progressive caloric restriction induces dose-dependent changes in myocardial triglyceride content and diastolic function in healthy men. J Clin Endocrinol Metab 93:497-503

16. van der Meer RW, Hammer S, Lamb HJ et al (2008) Effects of short-term high-fat, high-energy diet on hepatic and myocardial triglyceride content in healthy men. J Clin Endocrinol Metab 93:2702-2708

17. Moore MC, Satake S, Lautz M et al (2004) Nonesterified fatty acids and hepatic glucose metabolism in the conscious dog. Diabetes $53: 32-40$

18. Guiducci L, Burchielli S, Chubuchny Vet al (2011) Maternal and sex dependency of insulin resistance: longitudinal PET and echocardiography study from the healthy fetus to the adult minipig. J Nucl Med 52:1993-2000

19. Bickerton AS, Roberts R, Fielding BA et al (2007) Preferential uptake of dietary fatty acids in adipose tissue and muscle in the postprandial period. Diabetes 56:168-176

20. Henze E, Huang SC, Ratib O, Hoffman E, Phelps ME, Schelbert HR (1983) Measurements of regional tissue and blood-pool radiotracer concentrations from serial tomographic images of the heart. J Nucl Med 24:987-996

21. Dunn JT, Anthony K, Amiel SA, Marsden PK (2009) Correction for the effect of rising plasma glucose levels on quantification of MR(glc) with FDG-PET. J Cereb Blood Flow Metab 29:1059-1067

22. Patlak CS, Blasberg RG (1985) Graphical evaluation of blood-tobrain transfer constants from multiple-time uptake data. Generalizations. J Cereb Blood Flow Metab 5:584-590

23. Botker HE, Bottcher M, Schmitz O et al (1997) Glucose uptake and lumped constant variability in normal human hearts determined with [18F]fluorodeoxyglucose. J Nucl Cardiol 4:125-132

24. Ng CK, Soufer R, McNulty PH (1998) Effect of hyperinsulinemia on myocardial fluorine-18-FDG uptake. J Nucl Med 39:379-383

25. Iozzo P, Gastaldelli A, Jarvisalo MJ et al (2006) 18F-FDG assessment of glucose disposal and production rates during fasting and insulin stimulation: a validation study. J Nucl Med 47:1016-1022
26. Folch J, Lees M, Sloane Stanley GH (1957) A simple method for the isolation and purification of total lipides from animal tissues. J Biol Chem 226:497-509

27. Heath RB, Karpe F, Milne RW, Burdge GC, Wootton SA, Frayn KN (2003) Selective partitioning of dietary fatty acids into the VLDL TG pool in the early postprandial period. J Lipid Res 44:2065-2072

28. Evans K, Burdge GC, Wootton SA, Clark ML, Frayn KN (2002) Regulation of dietary fatty acid entrapment in subcutaneous adipose tissue and skeletal muscle. Diabetes 51:2684-2690

29. Knuuti MJ, Maki M, Yki-Jarvinen H et al (1995) The effect of insulin and FFA on myocardial glucose uptake. J Mol Cell Cardiol 27:1359-1367

30. Vitale GD, deKemp RA, Ruddy TD, Williams K, Beanlands RS (2001) Myocardial glucose utilization and optimization of (18)FFDG PET imaging in patients with non-insulin-dependent diabetes mellitus, coronary artery disease, and left ventricular dysfunction. J Nucl Med 42:1730-1736

31. Nuutila P, Knuuti MJ, Raitakari M et al (1994) Effect of antilipolysis on heart and skeletal muscle glucose uptake in overnight fasted humans. Am J Physiol 267:E941-E946

32. Tuunanen H, Engblom E, Naum A et al (2006) Free fatty acid depletion acutely decreases cardiac work and efficiency in cardiomyopathic heart failure. Circulation 114:2130-2137

33. Lehto HR, Parkka J, Borra R et al (2012) Effects of acute and oneweek fatty acid lowering on cardiac function and insulin sensitivity in relation with myocardial and muscle fat and adiponectin levels. J Clin Endocrinol Metab 97:3277-3284

34. Winhofer Y, Krssak M, Jankovic D et al (2012) Short-term hyperinsulinemia and hyperglycemia increase myocardial lipid content in normal subjects. Diabetes 61:1210-1216

35. Reingold JS, McGavock JM, Kaka S, Tillery T, Victor RG, Szczepaniak LS (2005) Determination of triglyceride in the human myocardium by magnetic resonance spectroscopy: reproducibility and sensitivity of the method. Am J Physiol Endocrinol Metab 289: E935-E939

36. Coggan AR, Kisrieva-Ware Z, Dence CS, Eisenbeis P, Gropler RJ, Herrero P (2009) Measurement of myocardial fatty acid esterification using [1-11C]palmitate and PET: comparison with direct measurements of myocardial triglyceride synthesis. J Nucl Cardiol 16:562-570

37. Rider OJ, Holloway CJ, Emmanuel Y, Bloch E, Clarke K, Neubauer S (2012) Increasing plasma free fatty acids in healthy subjects induces aortic distensibility changes seen in obesity. Circ Cardiovasc Imaging 5:367-375

38. Hammer S, Jonker JT, Lamb HJ et al (2008) Short-term hyperglycemic dysregulation in patients with type 1 diabetes does not change myocardial triglyceride content or myocardial function. Diabetes Care 31:1613-1614

39. Gerstein HC, Miller ME, Byington RP et al (2008) Effects of intensive glucose lowering in type 2 diabetes. N Engl J Med 358:2545-2559

40. Duckworth W, Abraira C, Moritz T et al (2009) Glucose control and vascular complications in veterans with type 2 diabetes. N Engl J Med 360:129-139 